\title{
دور السياسات العامة الرشيدة في تحقيق اهداف التنمية المستدامة المتيذة في
}

أ.م.د محمد علي حمود)"

المقدمة

شهدت العالم منذ نهايات القرن الماضي وبدايات القرن الواحد والعشرين تطورات دراماتيكية على صعيد الادبيات التنموية ، اذ تحولت التنمية من المفهوم التقليدي الذي اهتم بالنمو الاقتصادي الى رؤية جديدة هي رؤية التنمية البشرية ومن ثم الى التنمية المستدامة التي اعطت للتنمية البعد الانساني وجعلت من مشكلات واحتياجات البشر منطلق لها لتحويل الفرد والمجتمع الى مرحلة جديدة تضمن له له العيش الكريم وتحقق معه نسب مهمه من الحرية والثقافة والتعليم والصحة والنظافة والاكتفاء وغيرها هذا النطور ادى الى اعادة النظر بمفهوم التنمية ليكون مفهوما يندمج فيه الجانب الاقتصادي والاجتماعي وحتى الجانب السياسي.

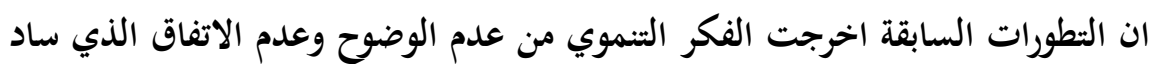
لفترات طويلة حول ماهية التنمية وما هو المقصود بها وهل هي تتضمن بعدا اقتصاديا فتط ام اقتصاديا واجتماعيا وهذا ادى الى تخبط في كثير من التجارب لتبرز لنا مفهوم

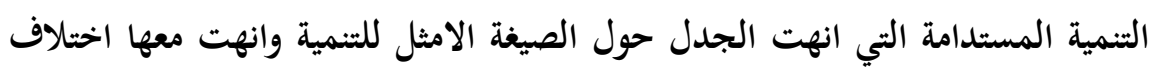

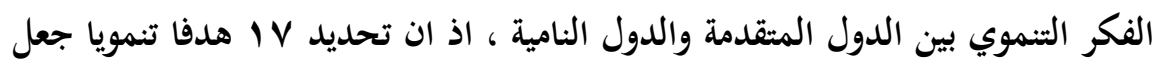
من الغاية التنموي واضحة المعالم ولا تحتاج الى اجتهاد او تفسير فكل الحكومات يمكن ان تتجه لتحقيق هذه الاهداف بغض النظر عن مستواها التنموي . 
ان تحقيق اهداف التنمية المستدامة يتطلب وجود بيئة مؤاتيه لتحقيقها وهذه البيئة تتضمن بعدا سياسيا واجتماعيا واقتصاديا ويتطلب وجود اداء حكومي فعال ،وتبرز اهمية

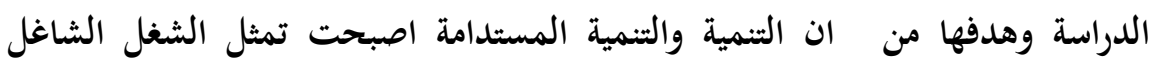
للمنظمات الدولية ولمؤسسات المجتمع الدولي والحكومات ومنظمات المجتمع المدني والاعلام ولا نجد اليوم أي فعالية دولية تهتم بالبلدان العالم ومشكلاته الا وتطرقت الى التنمية المستدامة من جهة اخرى فان هدف البحث يتضمن معالجة نقطة

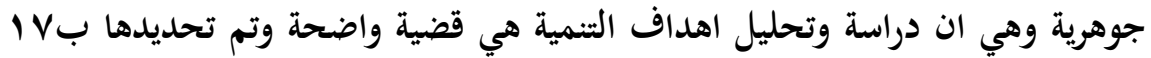

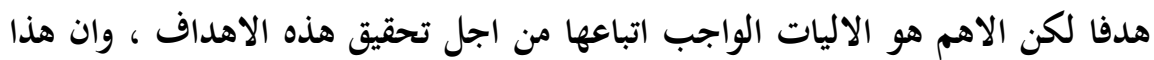

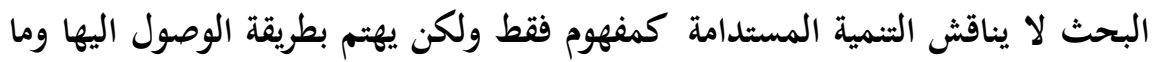
هي الطرق الواجب اتباعها لتحقيق ذلك. اشكالية البحث

تنطلق اشكالية البحث من فكرة اساسية هي ان هناك دول ذات ظروف متشابهة وتواجه نفس التحديات ويمكن ان تكون في نفس المستوى المعيشي او متقارب الى لى الى حد ما ولكن انقسمت هذه البلدان الى دول فاشلة وذات مستويات معيشية متدنية ودول اخرى ذات تجارب تنموية وناجحة. فما هي مسببات ذلك خاصة في بلدان

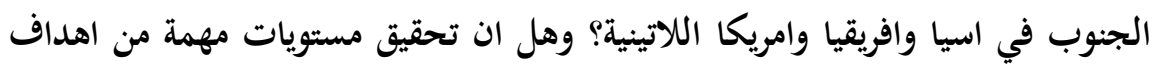
التنمية المستدامة قد ارتبط بخحائص محددة للسياسات العامة في هذه الدول؟ وهل كان الاداء الحكومي يتميز بأساليب في البلدان التي حققت مستويات من التنمية

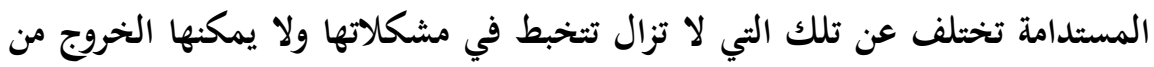
الواقع المتخلف فيها ؟ أمناه فرضية البحث البح المئ ان الدول التي استطاعت تحقيق مستويات متميزة من التعليم والصحة والدخل

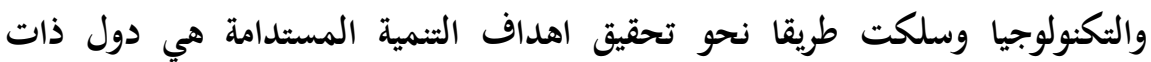
حكومات تبنت العقلانية والرشادة في سياساتها العامة ومن خلال هذه السياسات 
الرشيدة -العقلانية استطاعت تذليل كل التحديات التي واجهتها والقضاء على كل مظاهر التخلف ونقلت مجتمعاتها الى مصاف الدول المتطورة خاصة على صعيد مؤشرات التنمية البشرية واصبح لديها ديمومة في ادائها الحكومي من خلال سياسات ليست استهلاكية وانما سياسات تتضمن عوائد مادية ومعنوية تخدم المجتمع وتؤمن عمل الحكومة في مختلف القطاعات. وتعتمد الدراسة في تحليلاتها على المنهج النظمي الذي يرتبط بأربعة عناصر اساسية

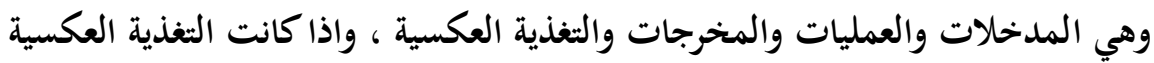

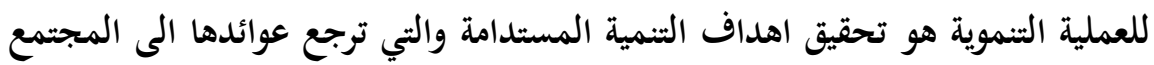
ومن اجل تحقيق مخرجات حكومية فعالة لا بد ان تتسم العمليات السمة العقلانية والرشيدة في عملية صنع القرار بناءً على مدخلات المجتمع المادية والمعنوية. ومن اجل اختبار هذه الفرضية سيتم تقسيم البحث الى ثلاث محاور اساسية هي: - معوقات التنمية والتحول الى اهداف التنمية المستدامة - - البعد التنموي لنظرية الاختيار الرشيد(العقلاني) - - فاعلية السياسات العامة الرشيدة في تحقيق التنمية المستدامة 1-معوقات التنمية والتحول الى اهداف التنمية المستدامة تعد عملية تحقيق التنمية من اكثر التحديات التي تواجه الدول وشعوبها وعلى الخصوص البلدان النامية او دول الجنوب ان صح التعبير فكثير من النظم السياسية المعاصرة فشلت في تحقيق الاهداف الثنموية رغم انها رفعت هذه الشعارات وبرمجتها كخطط سنوية وخمسية ومع ذلك لم تستطع نقل مجتماعاتها من الواقع المتخلف الذي

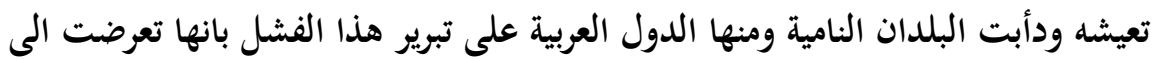
تحديات ومؤامرات داخلية وخارجية ودولية وهذا يمثل وهما حاولت هذه الحكومات

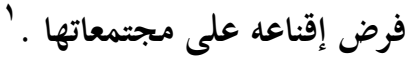
بالمقابل هناك دول استطاعت ان تحقق تجارب تنموية مهمة ونقلت مجتمعاتها الى واقع متطور وتنموي ويعتمد على التكنولوجيا ويحقق مستوى جيد من معايير التعليم والصحة 
والدخل والمشاركة ومن هذه الدول على سبيل المثال كوريا الجنوبية وماليزيا وسنغافوره والامارات .

$$
\text { 1- امعوقات ومبررات فشل التنمية في البلدان النامية }
$$

لا يوجد تفسير واحد لأسباب فشل الثنمية في الكثير من البلدان النامية فلكل منطقة

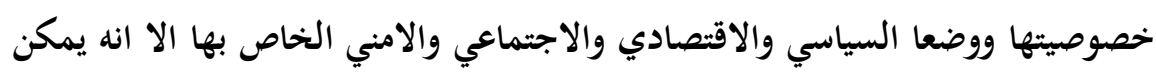
القول ان هناك وجهات نظر حول اهم معرقلات التجارب التنموية ،فقد نصت خطئ

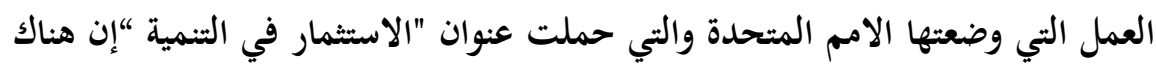
عدة اسباب لفشل التنمية يمكن اختصارها بالاتي: (الادارة السيئة المرتبطة بالفساد وسوء الخيارات التي تتعلق بالسياسات الاقتصادية والحرمان من حقوق الانئ الانسان ومشكلة الفقر).

$$
\text { من جهة اخرى هناك من يرى ان ابرز معوقات التّمية هي: }
$$

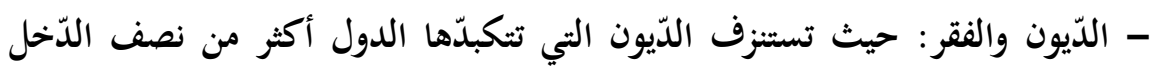

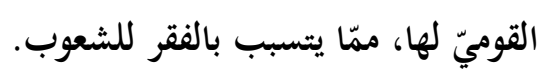

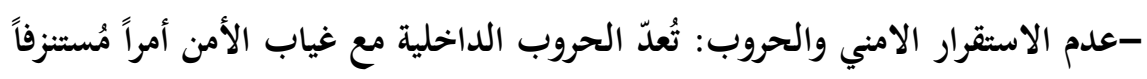
للأموال في الدّول التي تعاني من سباق التسلّح والحروب الداخلية.

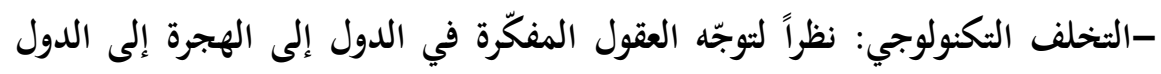
المتقدمة، ويعود الأمر بالسّلب على خطط التّمية.

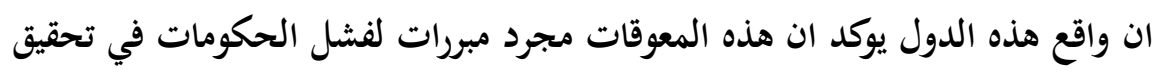
التنمية ومواجهة معوقاتها ولعل ذلك يرجع الى طريقة الحكم والفلسفة التي بنيت عليها

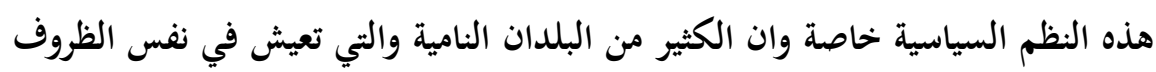
ونفس التحديات ولكنها نجحت في تحقيق مستويات تنموية متقدمة.

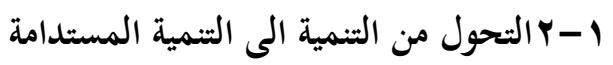


لقد اجابت (خطة التنمية المستدامة • • • التي تبنتها الامم المتحدة على تساؤل مهم حول حقيقة الخلط والتداخل بين الاهداف الانمائية للألفية وبين اهداف التنمية

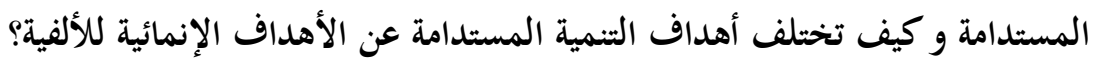

\section{ويمكن توضيح الاختلاف وفقا لرؤية الامم المتحدة بالاتي:؟}

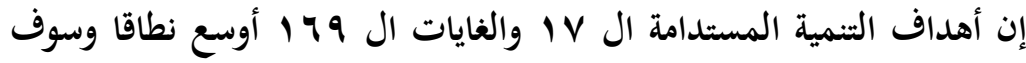
تذهب أبعد مما ذهبت إليه الأهداف الإنمائية للألفية من خحلال معالجة الأسباب الجذرية للفقر والحاجة العالمية للتنمية التي تعمل لصالح الجميع.

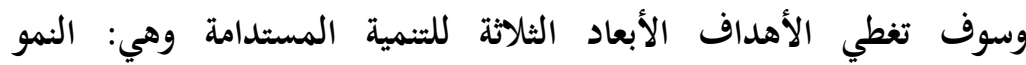
الاقتصادي والإدماج الاجتماعي وحماية البيئة؛.

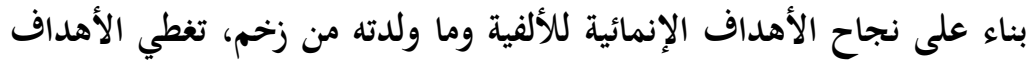

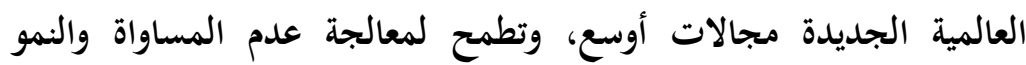
الاقتصادي والعمل اللائق والمدن والمستوطنات البشرية والتصنيع والمحيطات والنظم الإيكولوجية والطاقة وتغير المناخ والاستهلاك والإنتاج المستدامين والسلام والعدالة. الأهداف الجديدة عالمية وتنطبق على جميع البلدان، بينما كانت الأهداف الإنمائية للألفية مخصصة للعمل في البلدان النامية فقط. هناك سمة أساسية لأهداف التنمية المستدامة تتمثل في تركيزها القوي على لئل

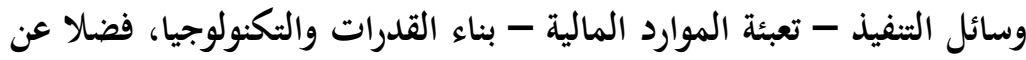

$$
\text { البيانات والمؤسسات. }
$$

تعترف الأهداف الجديدة بأن التعامل مع تغير المناخ أمر أساسي لتحقيق

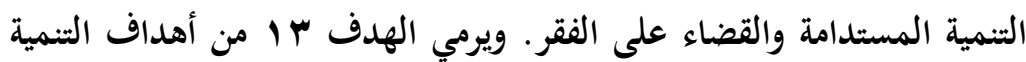
المستدامة إلى تشجيع اتخاذ إجراءات عاجلة لمكافحة تغير المناخ وآثاره. 
ان التحديات والالتزامات التي تم تحديدها في المؤتمرات الرئيسية للأمم المتحدة ومؤتمرات القمة مترابطة وتنطلب حلولا متكاملة، ولا بد في سبيل التصدي لها بفعالية من اتباع منهج جديد. فثمة اعتراف في مجال التنمية المستدامة بأن القضاء على الفقر بجميع صوره وأبعاده،

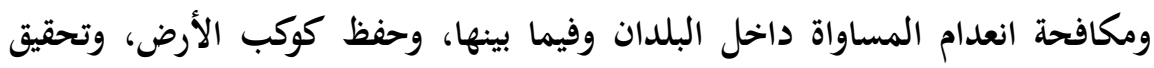

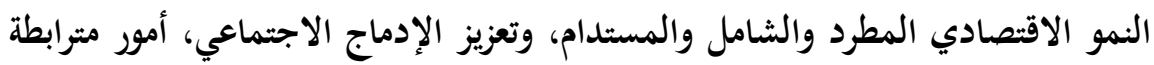
متصل بعضها ببعض ومترابطة. لقد مرت الكثير من الاعوام على الاتفاق على الأهداف الإنمائية للألفية، وهي أهداف بهده

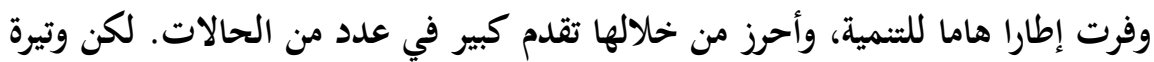
التقدم كانت متفاوتة، ولا سيما في أفريقيا وأقل البلدان نموا والبلدان النامية غير

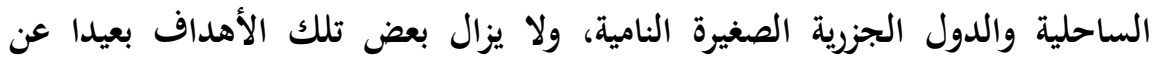
المسار الصحيح، لا سيما الأهداف المتعلقة بصحة الأمهات والمواليد والأطفال والصحة الإنجابية . غير أن الإطار الجديد الذي اعلنت عنه الامم المتحدة يتجاوز نطاقه بكثير نطاق الأهداف الإنمائية للألفية. فإلى جانب الأولويات الإنمائية القائمة مثل القضاء على القيل الفقر والاهتمام بالصحة والتعليم والأمن الغذائي والتغذية، يحدد هذا الإطار طائفة واسعة من الأهداف الاقتصادية والاجتماعية والبيئة. وهو يعد أيضا بإرساء مجتمعات

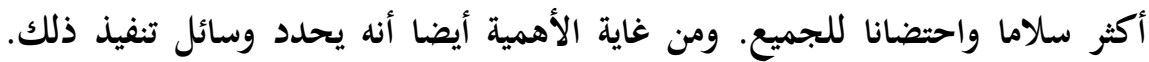
وترتبط جميع الأهداف والغايات الجديدة بروابط عميقة وتشترك كلها في عناصر عديدة، مما يعكس النهج المتكامل الذي قررت اتباعهه

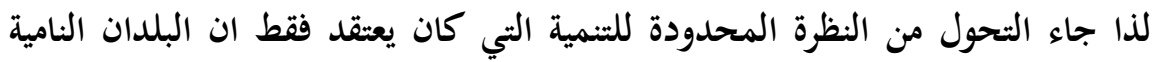
تفتقدها الى النظرة الجديدة التي طورت رؤية العالم للتنمية ليحولها الى فكرة عالمية وطموح لكل المجتمعات في الكرة الارضية بغض النظر عن مكان هذه الدولة او تلك لذا تحولت التنمية الى مستوى جديد اكثر نضوجا واهمية وبدء عصر جديد اجتمعت 
عليه كل الامم لتتحول التنمية الى البعد الجديد وهو بعد الاستدامة والذي تضمن وجود

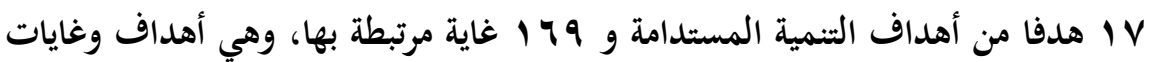
متكاملة وغير قابلة للتجزئة. ولم يسبق قط لزعماء العالم أن تعهدوا بالعمل معا وبذل المساعي المشتركة بشأن خطة سياساتية تملك هذا القدر

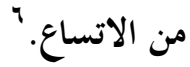
r-البعد التنموي لنظرية الاختيار الرشيد(العقلاني)

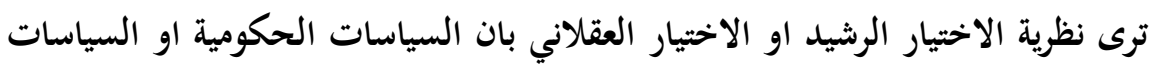

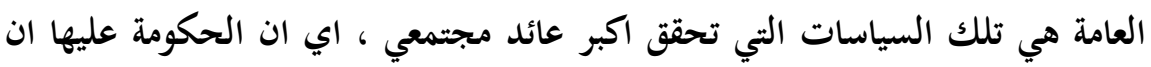

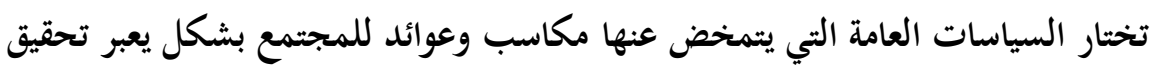

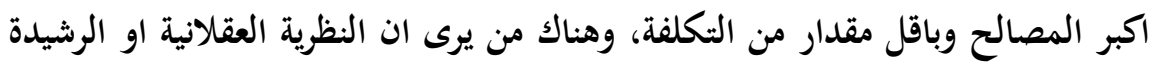
هي الافضل في السياسات العامة خاصة مع وجود اهداف ثابتة وواضحة المكا هو الحمال

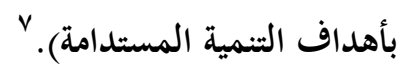
ان السياسات العامة تكون رشيدة او عقلانية عندما يكون هناك فرق ايجابي بين القيم التي تم تحقيقها وبين القيم التي تم التضحية بها وهذا لا يعني باي شكل من الاشكال ان تتم التضحية بقيم اجتماعية اساسية مقابل تعظيم القيم المادية فالرشادة والعقلانية تتضمن جميع قيم المجتمع الاقتصادية والسياسية والاجتماعية .

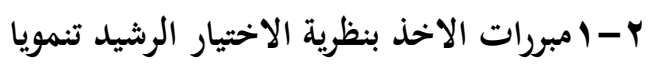
ويرى انصار هذه النظية ان هناك مبررات للأخذ بالعقلانية والرشادة في مجمل الفعل

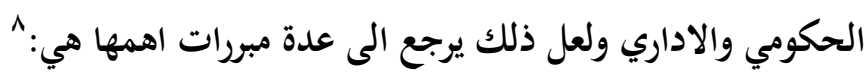

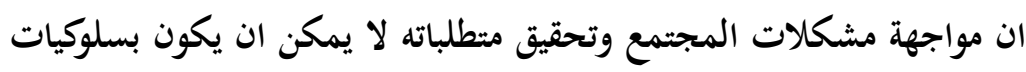

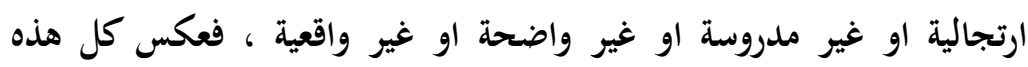
السلوكيات السابقة هي سلوكيات واقعية عقلانية ، فعند انتقادنا للأفعال الحكومية غير الفعالة فدائما ما نضع معايير الرشادة والعقلانية في اتخاذ القرار

$$
\text { وصنع السياسات. }
$$


ان التركيز على وجود عائد مادي وعنوي اكبر من التكلفة المادية والمعنوية سيحقق عدة جوانب اساسية ابرزها ان العوائد المتحققة ومنها العوائد المادية

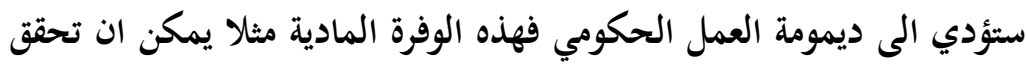
اضافة مستقبلية لسياسة جديدة وبالتالي تتمكن الحكومة من تحقيق عمل مستمر في قطاع مجتمعي معين وهذا يعني ان السياسات العامة ستكون

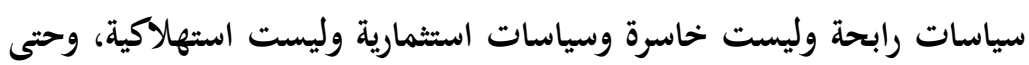

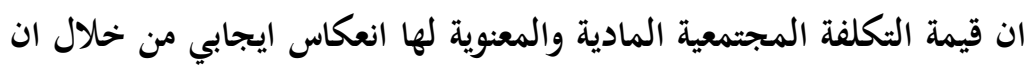
الفرد في المجتمع عندما يتحمل جزء من هذه التكلفة سواء كانت قليلة ام كبيرة فسيكون هذا الفرد جزء فاعل ومشارك في كل حيثيات هذه السياسة وعندما يجد اي خلل فلن يقبل به وسيكون رقيبا على هذه السياسات ولن

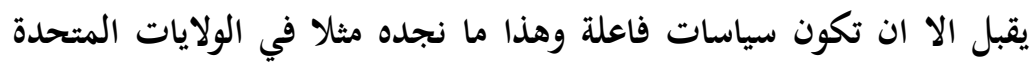

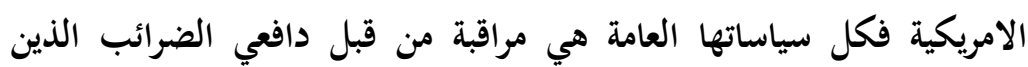
ينظرون مخرجات فعالة لهذه السياسات وبما تخدم مصالهم.

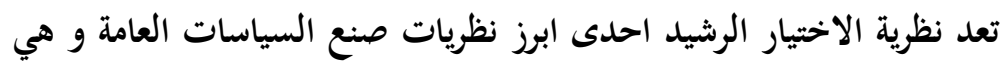

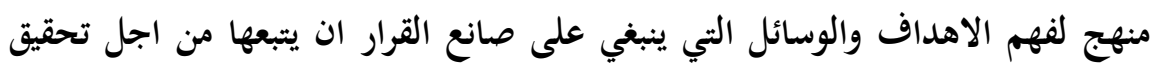

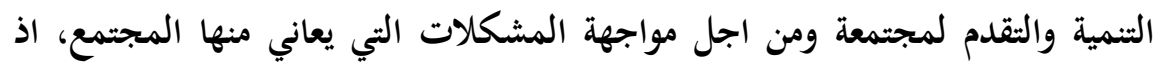

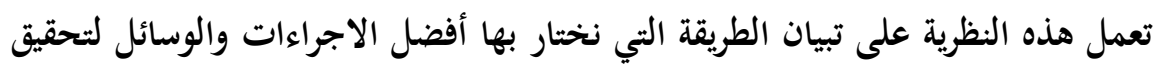

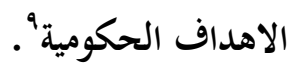

الاختيار الرشيد كنظرية تقوم على أساس تحديد السلوك الرشيد الذي يمكن اللاعب من الفوز، والمقصود سلوك صناع القرار، إذن الأفراد قد يتصرفون بشكل

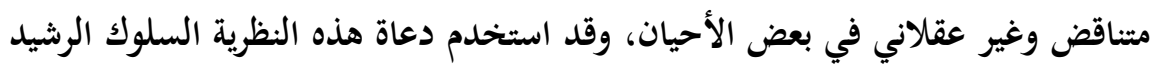
على أساس أنه الأكثر قدرة على جعل النظرية أصلح للتفسير، والسلوك الرشيد يعني بأن كل لاعب في السياسة يمتلك مجموعة من القيم والأهداف المحددة ويقرر سياسته طبقا لذلك بدون أخطاء. ويجب أن يتم ذلك على أسس رياضية واقعية ، ولهذا فِإن 
نظرية اللعبة هي طريقة للتحليل وهي أيضا دليل لاختيار أفضل طريق للعمل وترتكز هذه

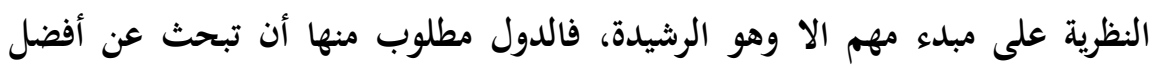

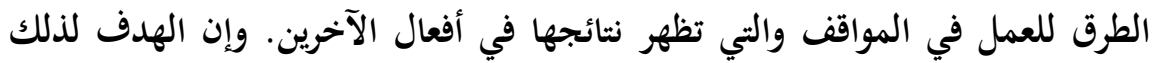
هو تحديد هذه الأفعال الرشيدة التي تستطيع أن تقود وتؤدي إلى قرارات ووسائل للعمل

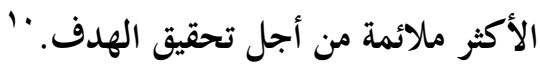

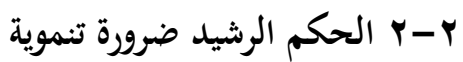

ذكر (جيمس غوستاف سبيث) المدير السابق لبرنامج الأمم المتحدة الإنمائي في

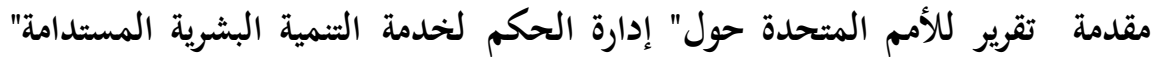
وهي وثيقة السياسات العامة لبرنامج الأمم المتحدة الإنمائي حول التنمية المستدامة اذ

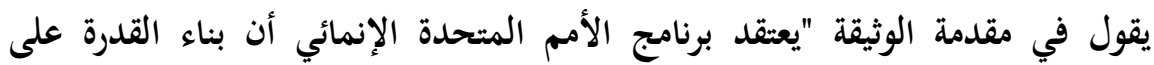

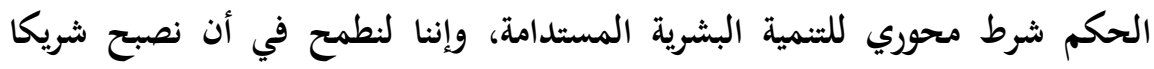

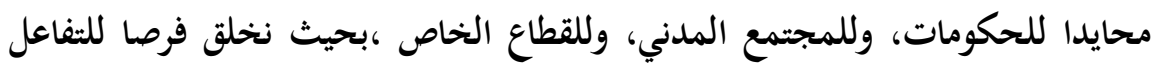
من أجل إيجاد حلول تتركز على الناس في الأجل الطويل". " توفر هذه الوثيقة المتعلقة بالسياسات العامة إطارا للتفاعلات التي يدخل التحل فيها برنامج الأمم المتحدة الإنمائي من أجل تسخير أساليب الحكم الرشيد لخدمة التنمية البشرية المستدامة. تواجه جميع المجتمعات تحديا يتمثل في إقامة نظام للحكم يعزز

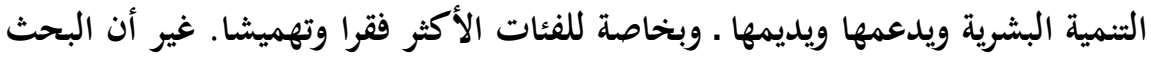
عن مفهوم محدد بوضوح للحكم لم يبدأ إلا مؤخرا جدا .

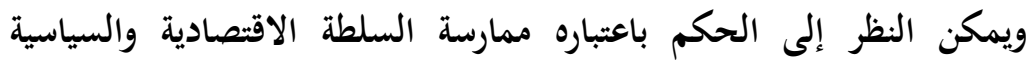

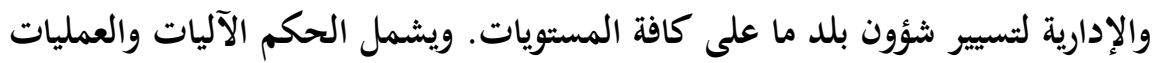
والمؤسسات التي يقوم من خلالها المواطنون والجماعات بالتعبير عن مصالحهم، وممارسة حقوقهم القانونية، والوفاء بالتزاماتهم، وتسوية خلافاتهم بـ ويتسم الحكم الرشيد بسمات عديدة، منها أن يقوم على المشاركة، وأن يتسم

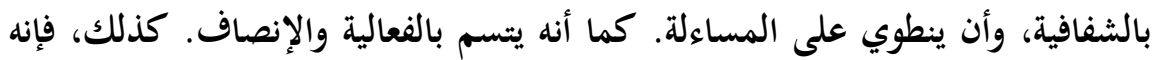


يعزز سيادة القانون. ويكفل الحكم الرشيد أن توضع الأولويات السياسية والاجتماعية

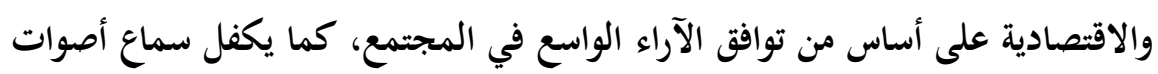
الفئات الأكثر فقرا والأكثر ضعفا في عملية صنع القرار المتعلق بتوزيع الموارد

$$
\text { الإنمائية. }
$$

وللحكم ثلاثة أضلع: الاقتصادي والسياسي والإداري، فالحكم الاقتصادي

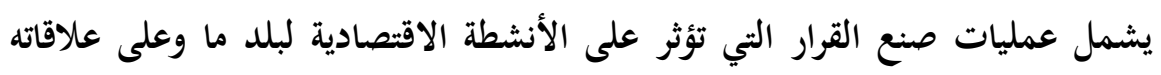

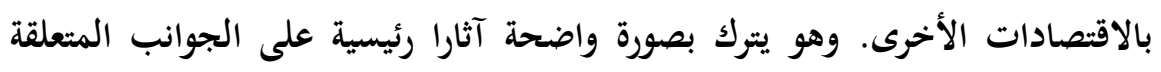

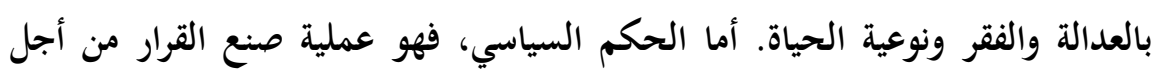

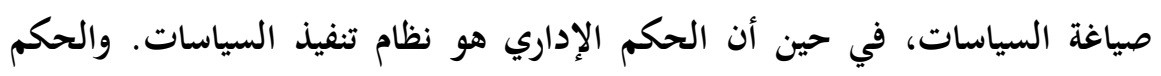

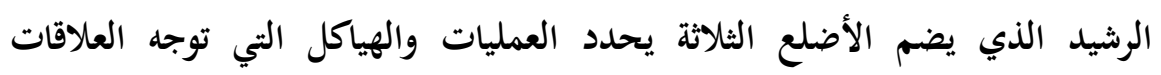
السياسية والاجتماعية ـ الاقتصادية.

والحكم يشمل الدولة، غير أنه يتجاوزها ليضم القطاع الخاص ومنظماتهات الاصنه

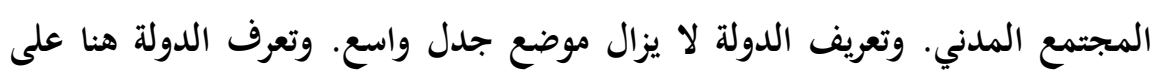

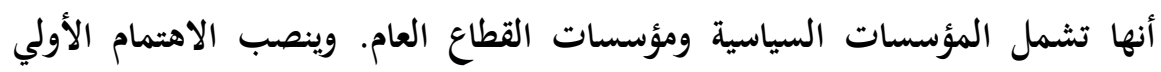
لبرنامج الأمم المتحدة الإنمائي مدى فعالية الدولة في مجال خدمة احتياجات شعبها ـ

ويغطي القطاع الخاص المؤسسات الخاصة (في مجالات التصنيع والتجارة والمصارف والتعاونيات وما إلى ذلك)، بالإضافة إلى القطاع غير الرسمي في السوق.

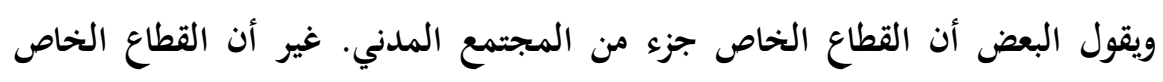

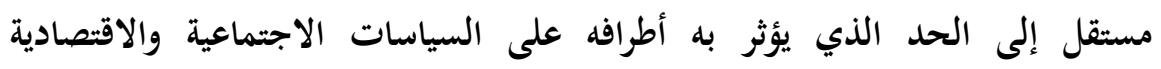
والسياسية بطرق تخلق بيئة مواتية بدرجة أكبر للسوق والمشاريع التجارية. 
أما المجتمع المدني، الذي يقع بين الفرد والدولة، فيضم الأفراد والجماعات

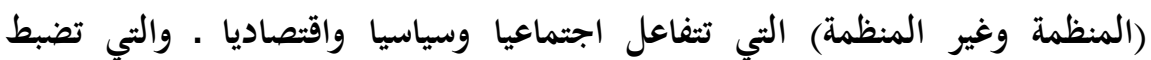
تفاعلاتها القواعد والقوانين الرسمية وغير الرسمية.

ومنظمات المجتمع المدني هي مجموعة الروابط الجمعيات التي ينظم بهات المجتمع نفسه بصورة طوعية. وهي تشمل النقابات؛ والمنظمات غير الحكومية؛

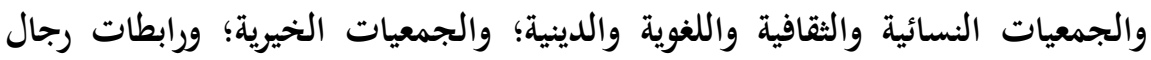
الأعمال؛ والنوادي الاجتماعية والرياضية؛ والتعاونيات ومنظمات تنمية المجتمعات المحلية؛ وجماعات البيئة؛ والروابط المهنية؛ والمؤسسات الأكاديمية ومؤسسات الأبحاث السياسية؛ ووسائل الإعلام. وتدخل فيها أيضا الأحزاب السياسية، وإن كانت تعد جزءا من المجتمع المدني ومن الدولة على حد سواء في حال تمثيلها في البرلمان.

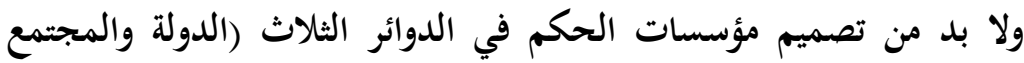
المدني والقطاع الخاص) على النحو الذي تسهم به في التنمية البشرية المستدامة من

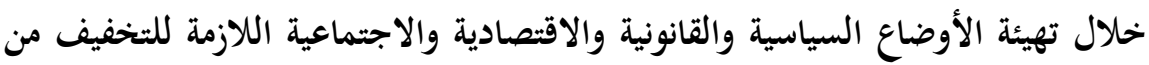
حدة الفقر وخلق الوظائف وحماية البيئة والنهوض بالمرأة؛ . وقد كتب الكثير عن سمات الحكومة الكفؤة والأعمال الثجارية الناجحة ومنظمات المجتمع المدني الفعالة، غير أنه يظل من الصعب تحديد سمات الحكم

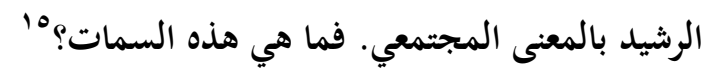

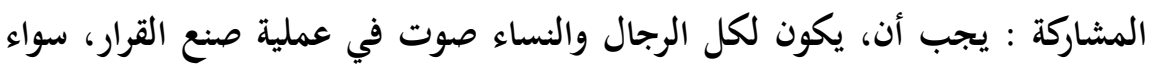
بصورة مباشرة أو من خلال مؤسسات وسيطة شرعية تمثل مصالحهم. وتسند هذه

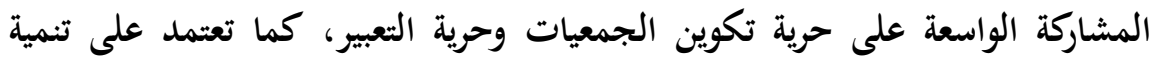
القدرات على المشاركة البناءة. سيادة القانون: يجب أن تتسم الأطر القانونية بالعدالة، ولا بد من توخي الحياد في إنفاذها، وبخاصة القوانين المتعلقة بحقوق الانسان. 
الشفافية: تأسس الشفافية على حرية تدفق المعلومات. فالعمليات والمؤسسات

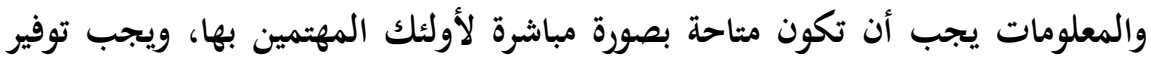

$$
\text { المعلومات الكافية لفهم تلك العمليات والمؤسسات ورصدها. }
$$

الاستجابة: يجب أن تسعى المؤسسات وتوجه العمليات إلى خدمة جميع أصحاب

المصلحة.

التوجيه نحو بناء توافق الآراء ـ يتوسط الحكم الرشيد المصالح المختلفة للوصول إلى

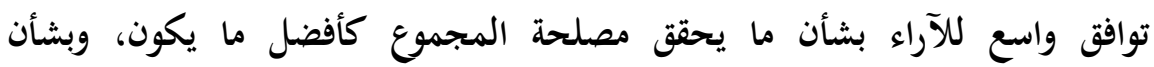
السياسات والإجراءات حيثما يكون ذلك ممكنا.

الإنصاف: يجب أن تتاح لجميع الرجال والنساء الفرصة لتحسين رفاههم أو الحفاظ

عليه.

الفاعلية والكفاءة : ينبغي أن تسفر العمليات والمؤسسات عن نتائج تلبي الاحتياجات مع تحقيق أفضل استخدام للموارد.

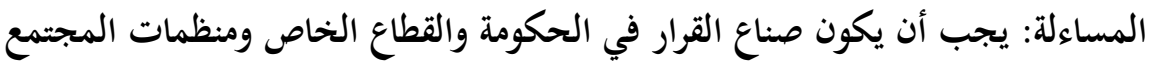

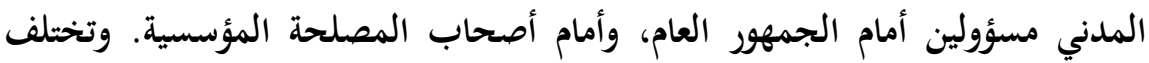

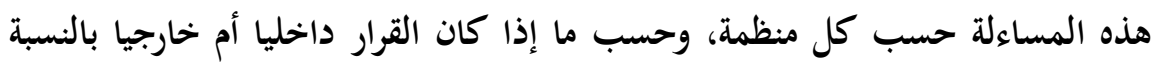
للمنظمة .

الرؤية الاستراتيجية: يجب أن يمتلك القادة والجمهور العام منظورا عريضا وطويل الأجل

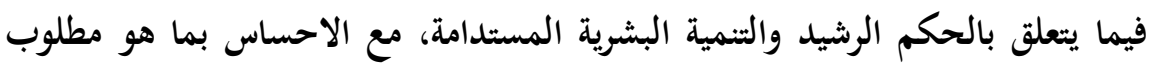

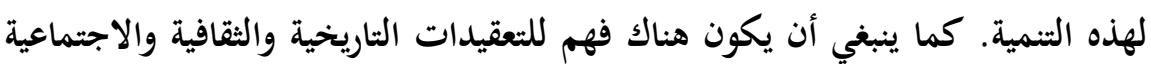
التي يتشكل وسطها ذلك المنظور" 14 وهذه السمات تترابط ويعزز بعضها البعض، بحيث لا يمكن أن يوجد أي منها بمفرده.

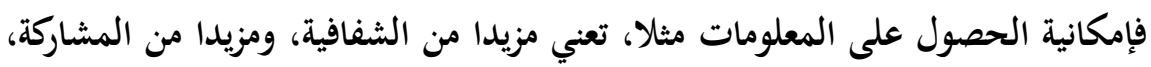
ومزيدا من فاعلية صنع القرار. كما أن اتساع نطاق المشاركة يسهم في كل من من تبادل 
المعلومات اللازمة لفاعلية صنع القرار وفي شرعية تلك القرارات. والشرعية بدورها تعني فاعلية التنفيذ، وتشجع على المزيد من المشاركة. كذلك، فإن استجابة المؤسسات

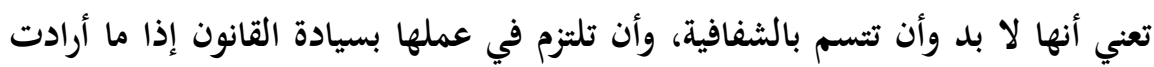
أن تكون منصفة.

مما تقدم ان الامم المتحدة ركزت من تسعينيات القرن العشرين على اهمية الحكم الرشيد في تحقيق التنمية المستدامة وبسبب التطورات التي طرئت على التى التنمية المستدامة من حيث الاهداف والاليات بدء التركيز على تفاصيل ادق وجزيئيات اهم ومن هنا بدء الاهتمام بضرورة تبني العقلانية والسياسات الرشيدة من اجل زيادة فئل فاعلية الحكومات واجهزة الدولة في طريق تحقيق اهداف التنمية المستدامة. ب-فاعلية السياسات العامة الرشيدة في تحقيق التنمية المستدامة تؤكد وجهة نظر الامم المتحدة لتحديات التنمية إخفاق الكثير من البلدان النامية ومنها المنطقة العربية في ترجمة ثرواتها المادية إلى تحسينات مقابلة في الرفاه الإنساني،

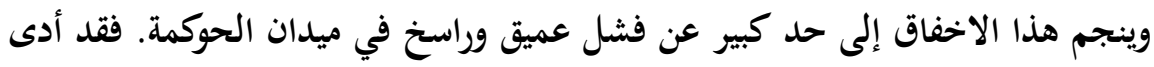
تزايد حصانة النخب السياسية والاقتصادية والإدارية وإفلاتها من العقاب، في في سيداق فيداق حريات مقيدة وآليات مساءلة هزيلة، إلى تقويض أسس كل نمو اقتصادي منصف وشامل للجميع. هناك الحاجة إلى اللعب السياسي النزيه و الرأي المسموع، فبدونهما لن يكون النمو كافياً لتحقيق التنمية .

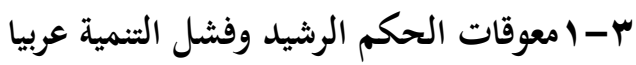

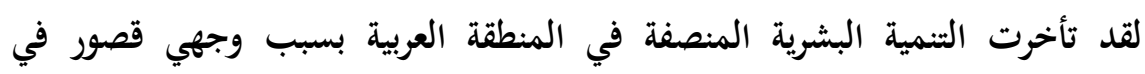

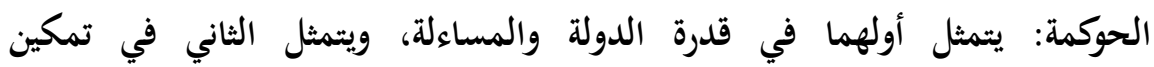
المجتمعات. وتشير الساعات الطويلة من المناقشات العامة التي رافقت الربيع العربي إلى أن الشعوب العربية تسعى إلى: 1- تعزيز شرعية القيادة من خحلال انتخابات حرة ونزيهة في جو من حرية التعبير والتنظيم. 
r- سياسات متجاوبة، استيعابية ومنصفة، تستهدي بشراكات شاملة بين الدولة

والمجتمع.

ץ- آليات فعالة لمناهضة الفساد، ورصد اجتماعي للسياسات العامة يتم تمكينه

عن طريق تيسير الوصول إلى المعلومات.

ع- استقلال السلطة القضائية.

فليس مدار الثنمية ومبتغاها خلق الثروة فحسب، وإنما أيضاً توزيع الثروة والمشاركة.

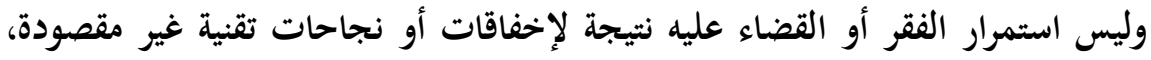
وإنما ننيجة لخيارات سياسية. فلمعالجة الفقر بصورة فعالة، يجب أن تضطلع الدول بدور قيادي في توسيع القدرات والحريات، وفي تمكين الناس. فأجنده الحوكمة الديمقراطية تستلزم إعطاء اهتمام خاص لاحترام حقوق الشعب وضمان كرامته، وهذا التحليل يؤكد بالمحصلة ان السياسات العامة التي هي حصيلة تفاعل النظام السياسي

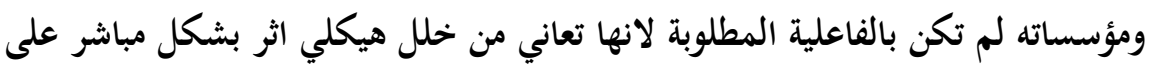
مخرجاتها التي لم تحقق التنمية ولم توجه الدولة نحو الاهداف الحقيقية للتنمية

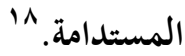
"وقد جاء في مؤتمر الامم المتحدة الثالث لتمويل التنمية والمنعقد في اثيوبيا والذي

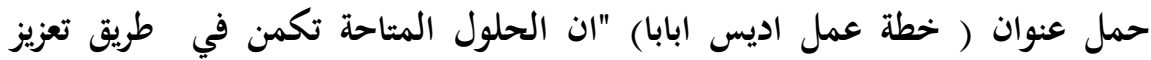
السياسات العامة والأطر التنظيمية والتمويل على جميع المستويات، وإطلاق العنان لقدرة الأشخاص والقطاع الخاص على إحداث التغيير، والتحفيز على إجراء تغييرات في

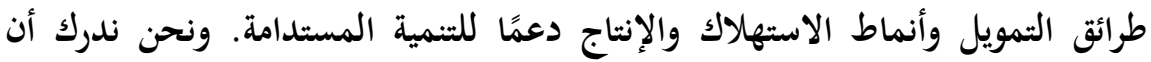
توفير الحوافز الملائمة، وتعزيز الأطر السياساتية والتنظيمية الوطنية والدولية وتحسين اتّساقها، والاستفادة من إمكانات العلوم والتكنولوجيا والابتكار، وسد الفجوات في التهري مجال التكنولوجيا، وتكثيف بناء القدرات على جميع المستويات هي عناصر أساسية لتحقيق التحوّل نحو التنمية المستدامة والقضاء على الفقر. ونعيد تأكيد أهمية الحرية،

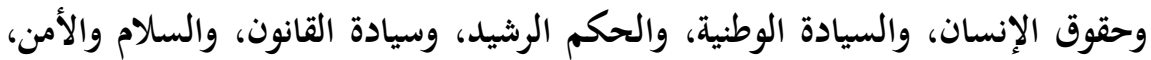


ومكافحة الفساد على كافة المستويات وبجميع أشكاله، والمؤسسات الديمقراطية الفعالة الخاضعة للمساءلة والش املة للجميع على كل من الصعيد دون الوطني والوطني

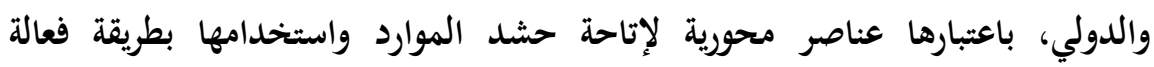

ب-Y بخائص السياسات العامة التنموية الرشيدة أن تحقيق التكامل اللازم بين السياسات العامة الرشيدة والفعالة يستدعي التحول عن وضع سياسات قطاعية منفصلة، ويقتضي العمل بالاستناد إلى الترابط الأفقي بين القطاعات والصلات العمودية للعمل بين مختلف مستويات الحكومة، ومن المهم اتباع

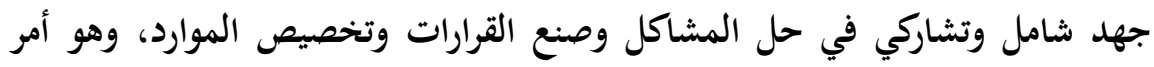

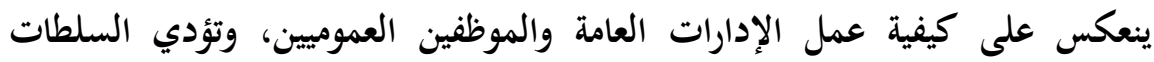
المحلية دورا حاسما في تنفيذ أهداف التنمية المستدامة، لذا ينبغي للبلدان أن تعزز الجهود الرامية إلى تشجيع التكامل السياساتي بين مستويات الحكومة ووضع أطر وطنية أكثر متانة تتيح تقاسم المسؤوليات والموارد في إطار السعي لتحقيق الأهداف إنى

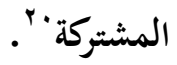

اصبح هناك تأكيد متزايد لتتبوأ السياسات العامة الرشيدة والعقلانية دورا مهما في تعبئة الموارد الوطنية واستخدامها بصورة فعالة، على أساس مبدأ رشيد يحقق وفرة مادية ومعنوية وبالنسبة لكافة البلدان، بما يشمل تنفيذ أهداف التنمية المستدامة. وتأسيسًا على التوجه الجديد للعديد من البلدان ، فإن هناك دعوات متواصلة لبلورة سياسات

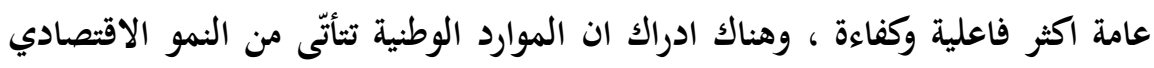
في المقام الأول، مدعومًا ببيئة مؤاتيه على جميع المستويات وخاصة الارادة السياسية، ولتنفيذ أهداف التنمية المستدامة، يلزم اعتماد سياسات اجتماعية وبيئية واقتصادية سديدة، بما فيها السياسات الضريبية الموجهة للتقلبات الدورية، وتوفير فسحة مالية كافية، وإرساء الحكم الرشيد على جميع المستويات ، وإنشاء مؤسسات ديمقراطية

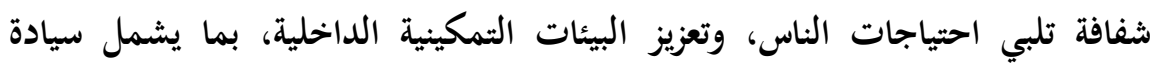


القانون، ومكافحة الفساد على كافة المستويات وبجميع أشكاله. ويؤدي المجتمع

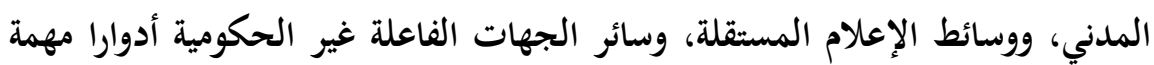

أيضا.

اصبحت العقلانية والرشادة مبدئ مهم في كل مفاصل ادارة الدولة ولعل كل الاشارات السابقة من المؤتمرات والندوات والاجتماعات التي تتعلق بالتنمية لم تخلو البيانات الختامية ولا التقارير الدورية عن التأكيد على مبدئ وجود سياسات عامة فعالة واهمية بلورة حكم رشيد يحقق سياسات عقلانية تقود الى الوصول الى اهداف التنمية

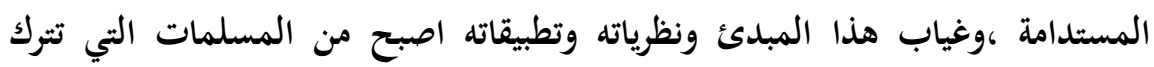

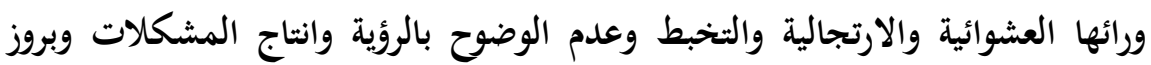
ازمات متعددة تواجه المجتمع والدولة.

أفضى التصور المتعلق بالمكونات الأساسية للسياسات العامة العقلانية والرشيدة في داخل المجتمع إلى فهم و إدراك جملة الخصائص الأساسية يجب ان تتميز بها كل الاجراءات الحكومية والفعل الاداري والسياسي التي يمكن ان يحقق التنمية المستدامة

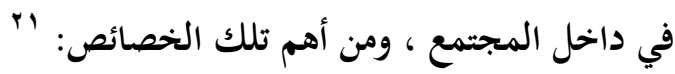
- - المشاركة: شريطة أن تكون هذه المشاركة منظمة وواقعية وفعالة ، وهذا ما

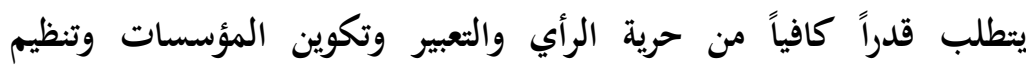
المجتمع المدني بحيث يضطلع بدوره في هذه العملية. - - ميادة القانون: إن السياسات الحكومية الرشيدة تنطلب أطراً قانونية عادلة يتم

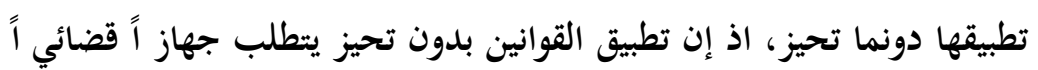

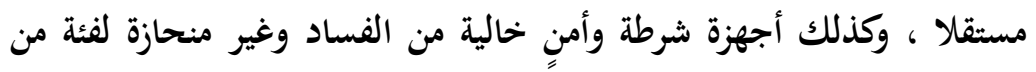
فئات المجتمع دون أخرى. - - - الشفافية: إن الشفافية تعني في شكلها العام أن القرارات التي يتم اتخاذها في

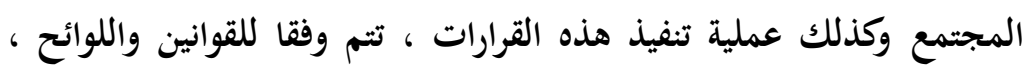


كما تعنى الشفافية أيضا ضرورة توفر المعلومات بحرية للجميع ، وأن يكون من حق كل فرد الوصول إليها بشكل كامل. - - الاستجابة: إن السياسات العامة الرشيدة تنطلب أن تقوم جميع المؤسسات

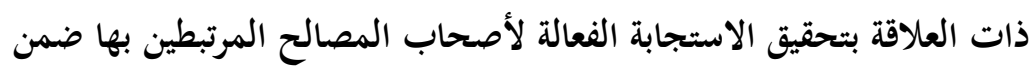

$$
\text { إطار زمني معقول. }
$$

- - التوافق: حيث أنه يوجد العديد من الأطراف في المجتمع يسعى كل منها نحو

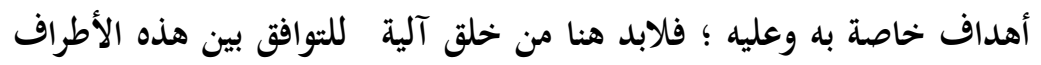
المختلفة بحيث يسعى الجميع إلى تحقيق أهداف المجتمع وتغليبها قدر

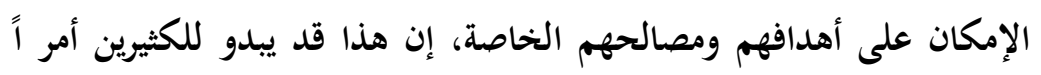

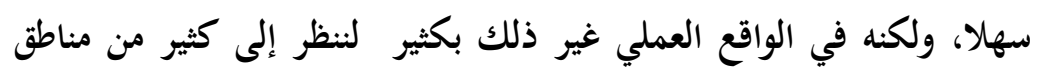

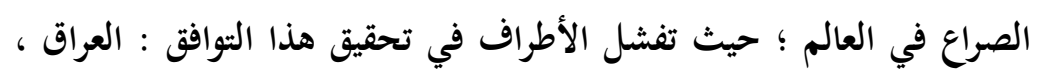

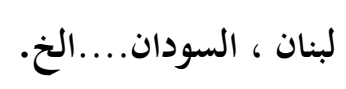

- - العدالة والمساواة: إن تقدم أي مجتمع إنما يعتمد على مدى شعور أفراده بأن

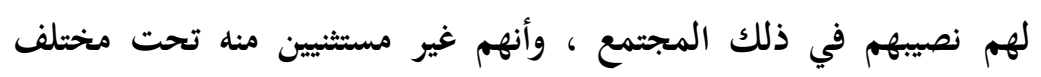

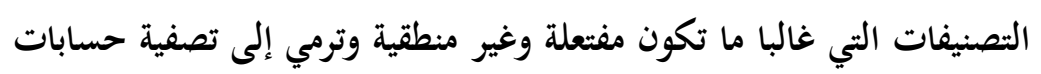

$$
\text { خاصة بين مختلف الأطراف. }
$$

- الكفاءة والكفاية: إن السياسات الرشيدة تكون فاعلة عندما تحقق المؤسسات

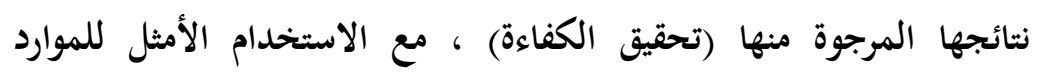

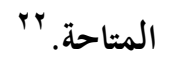

ورغم اهمية الحكم الرشيد في تحقيق اهداف التنمية المستدامة الا ان صفة

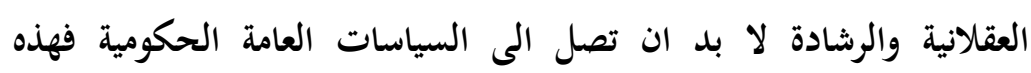
السياسات هي التي ستوجه العمل الحكومي وتقود الدولة في مضمار التنمية ولا بد من القول ان فشل التجارب التنموية فب الكثير من البلدان النامية 
ومنها البلدان العربية كان وراءه سياسات عامة تتصف بالارتجالية وعدم الوضوح والتخبط وان كانت هذه السياسات في اتجاهها العام هي سياسات

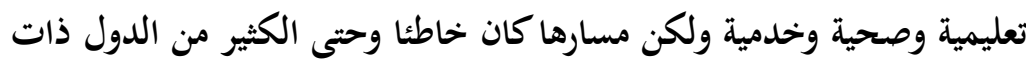

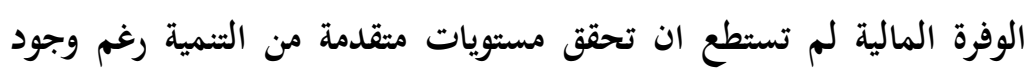

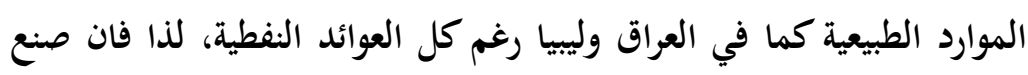
السياسات العامة وفقا لمعيار الرشادة والعقلانية ورغم كل الانتقادات لهذه

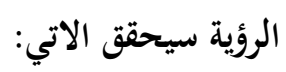

ستصنع السياسات العامة من اجل تحقيق عوائد مادية مهمة ستكون اداة

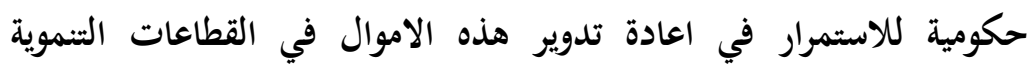

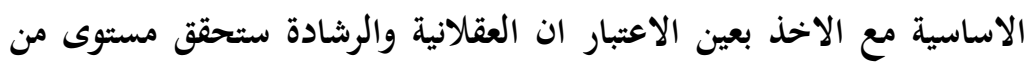

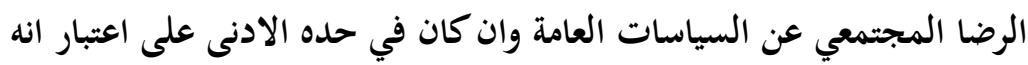
يتضمن تكاليف مادية قد يشترك المجتمع في تحقيقها. ان السياسات العامة الرشيدة والعقلانية ستؤدي الى اشراك المجتمع ليس في في لئي

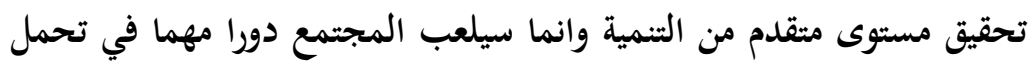

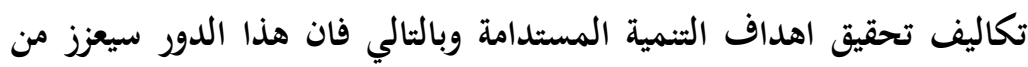
دوره الرقابي وستكون مشاركته ايجابية وليس مجرد طرف متلقي. ان عقلانية ورشادة السياسات العامة ستؤدي الى تكاليف مادية (كبيرة او قليلة) تفرض على المجتمعات مما يعني ان افراد المجتمع بكل مؤسساتهم

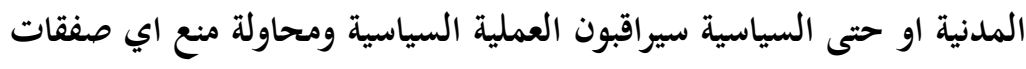

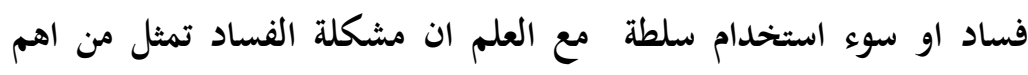
معرقلات تحقيق اهداف التنمية المستدامة. ان العقلانية والرشادة بطبيعتها تنزع نحو الاهتمام بتحقيق الاستقرار السياسي الهدي

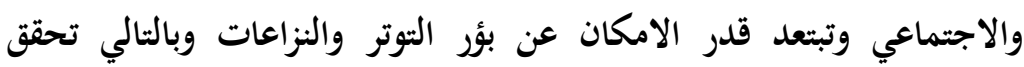
قاعدة من الاستقرار المطلوب في مراحل التنمية المستدامة المختلفة. 
ان صنع السياسات العامة وفقا للمبدء العقلاني والرشيد سيؤدي الى طمأنة

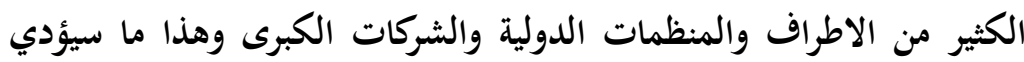
الى توفر بيئة مستقطبة للاستثمارات الخارجية وهذا يمثل مصدر تمويل للتنمية حتى عند اكثر الدول غنى. الخاتمة :

دخلت البلدان النامية مرحلة تنموية جديدة الا وهي مرحلة التنمية المستدامة التي

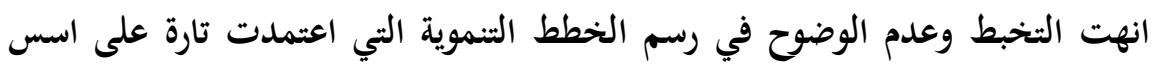
التنمية الاقتصادية واخرى على اسس التنمية الاجتماعية وغيرها ورغم كل المحاولات

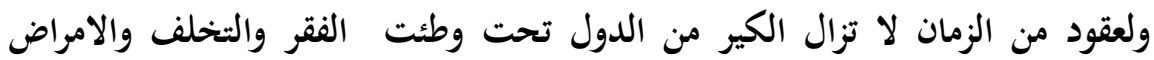
والاوبئة وعدم الاستقرار وتفشي مظاهر الفساد التي تمثل الضربة الاقوى والاخطر لمشاريع التنمية. لقد توجهت الجهود الدولية الى انهاء النقاش حول صيغ واشكال التنمية المطلوبة لتضع سبعة عشر هدفا مثلت الصيغة الافضل والارقى للتنمية مع بعدا اساسيا الا وهو بعد الاستدامة، ولكن الوضوح والاستقرار في طبيعة التنمية المطلوبة لا يعني تحقيقها ،فرغم الجهود المبذولة من العديد من الدول فلا تزال هناك عقبات مهمة في طريق الوصول

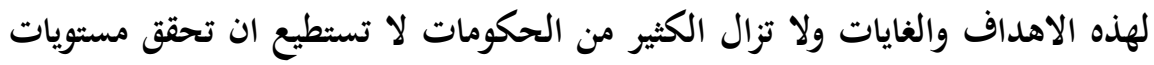

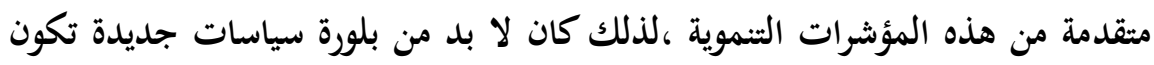
اكثر فاعلية واكثر قدرة على مسايرة متطلبات تحقيق التنمية المستدامة.

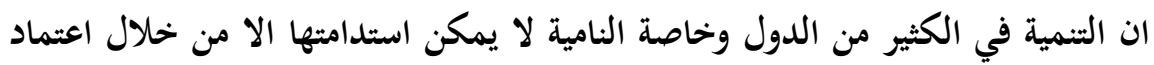

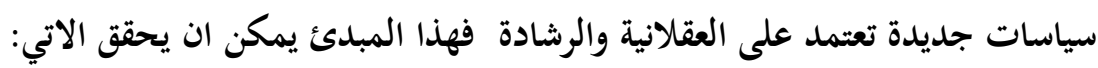
ستصنع السياسات العامة من اجل تحقيق عوائد مادية مهمة ستكون اداة حكومية للاستمرار في اعادة تدوير هذه الاموال في القطاعات التنموية الاساسية 
ان السياسات العامة الرشيدة والعقلانية ستجعل من المجتمع لاعبا مهما في تحمل

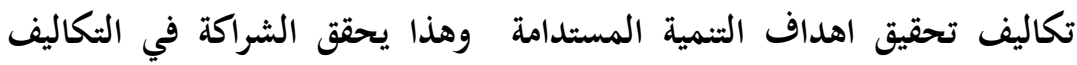

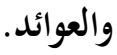

ان عقلانية ورشادة السياسات العامة ستدفع المجتمع لمراقبة العملية السياسية ومحاولة منع اي صفقات فساد او سوء استخدام سلطة . ان العقلانية والرشادة بطبيعتها تنزع نحو الاهتمام بتحقيق الاستقرار السياسي وبالثالي تحقق قاعدة من الاستقرار المطلوب في مراحل التنمية المستدامة

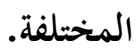
الهوامش:

' - لتفاصيل اكثر حول تحديات التنمية في البلدان العربية يمكن الرجوع الى برنامج الامم المتحدة الانمائي: تقر ير التربية

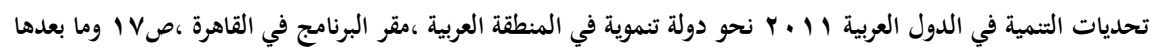

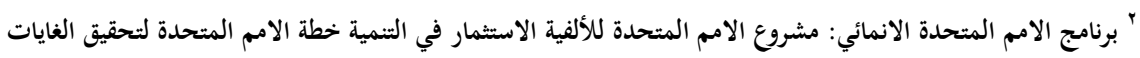

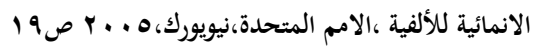

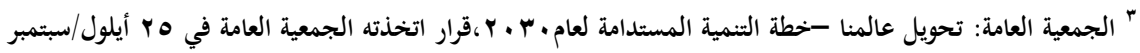

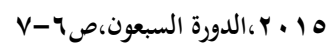
؛ للتفاصيل حول مجمل اهداف وغايات التنمية المستدامة يمكن الرجوع الى تقرير اهداف التنمية المستدامة

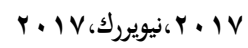

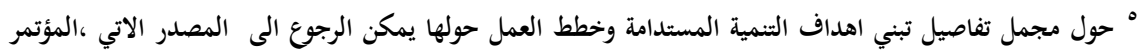

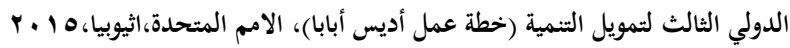

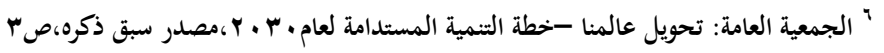

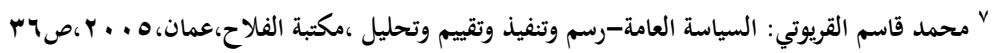

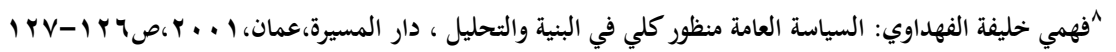

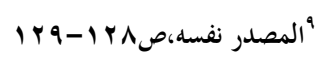

” يمكن الرجوع حول ارتباط بين العقلانية وبين السياسات العامة وحول الرؤية غير المتفائلة لهذا الربط يمكن الرجوع الى الى الى الريل

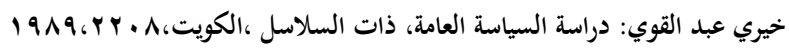

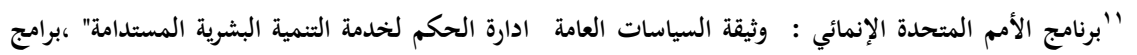




$$
\begin{aligned}
& \text { "'المصدر نفسه،ص } \\
& \text { "'المصدر نفسه،ص 9-1. }
\end{aligned}
$$

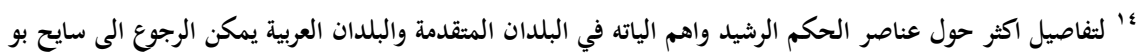

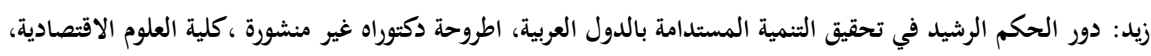

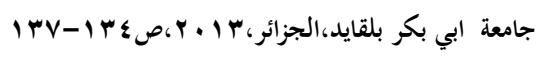

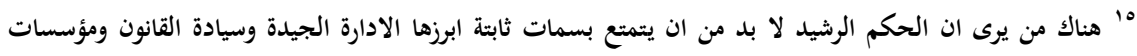

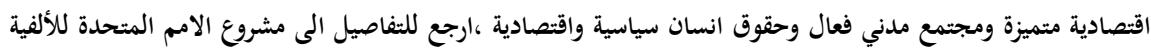

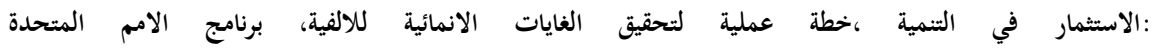

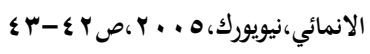

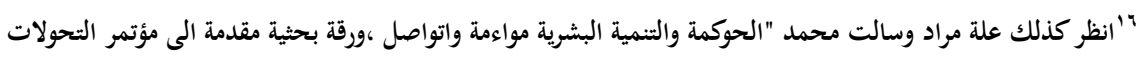

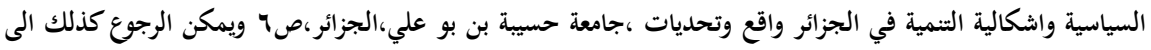

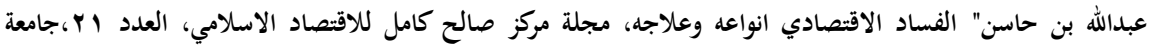

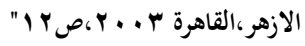

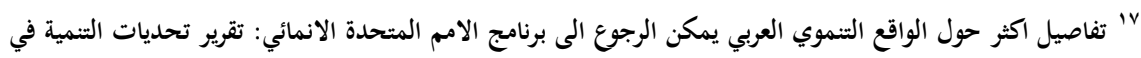

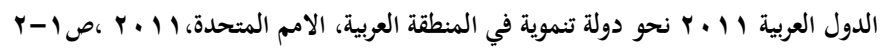

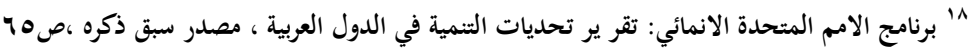

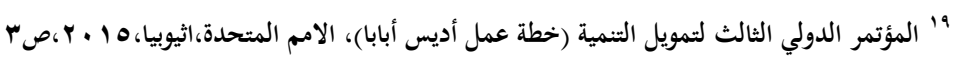

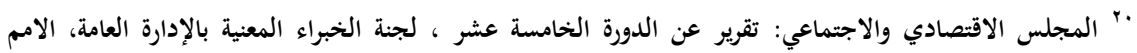

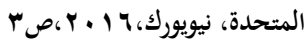
" أبوبكر مصطفى بعير، أنس ابوبكر بعيرة " لا تنمية مستدامة بدون إدارة قوامة "، مؤتمر التنمية المستدامة في ليبيا،

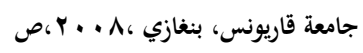

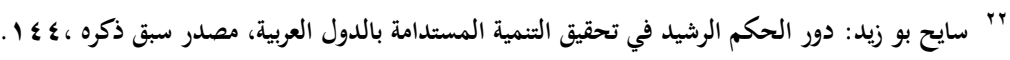


